\title{
MicroRNA-301a targets WNT1 to suppress cell proliferation and migration and enhance radiosensitivity in esophageal cancer cells
}

\author{
HUAFANG SU ${ }^{1,2}$, YOUYI WU ${ }^{3}$, YA FANG $^{2}$, LANXIAO SHEN $^{2}$, ZHENGHUA FEI $^{2}$, \\ CONGYING XIE ${ }^{2 *}$ and MING $\mathrm{CHEN}^{1,4^{*}}$ \\ ${ }^{1}$ Department of Radiotherapy and Oncology, The Second Affiliated Hospital of Soochow University, Suzhou, Jiangsu 215004; \\ ${ }^{2}$ Department of Radiation Oncology and Chemotherapy, The First Affiliated Hospital of Wenzhou Medical University; \\ ${ }^{3}$ Department of Radiation Oncology, The Third Affiliated Hospital of Wenzhou Medical University, \\ Wenzhou, Zhejiang 325000; ${ }^{4}$ Department of Radiation Oncology, Zhejiang Cancer Hospital, \\ Hangzhou, Zhejiang 310022, P.R. China
}

Received May 13, 2018; Accepted October 8, 2018

DOI: $10.3892 /$ or.2018.6799

\begin{abstract}
Esophageal cancer (EC) is one of the leading causes of death among malignancies. Radiotherapy for esophageal squamous cell carcinoma (ESCC) patients is limited by resistance to ionizing radiation (IR). An increasing body of evidence has demonstrated that aberrant expression of microRNA-301a (miR-301a) contributes to cancer progression and sensitivity to radiation. The aim of the present study was to investigate the exact functions and potential mechanisms of miR-301a in ESCC radioresistance. Initially, the miR-301a-transfected radioresistant ESCC cells KYSE-150R exhibited a decreased proliferation rate, and enhanced radiosensitivity and migration, whereas downregulation of miR-301a in radiosensitive KYSE-150 cells produced the opposite results. miR-301a regulates WNT1 expression at both the mRNA and protein levels. Furthermore, dual-luciferase reporter assays revealed that WNT1 was a target gene of miR-301a. In addition, the expression of miR-301a markedly affected the expression of $\mathrm{Wnt} / \beta$-catenin-related proteins such as $\beta$-catenin and cyclin D1. Finally, overexpression of miR-301a inhibited epithelial-mesenchymal transition (EMT) conversion by
\end{abstract}

Correspondence to: Dr Ming Chen, Department of Radiotherapy and Oncology, The Second Affiliated Hospital of Soochow University, 1055 Sanxiang Road, Suzhou, Jiangsu 215004, P.R. China E-mail: chenming@zjcc.org.cn

Dr Congying Xie, Department of Radiation Oncology and Chemotherapy, The First Affiliated Hospital of Wenzhou Medical University, 2 Fuxue Lane, Wenzhou, Zhejiang 325000, P.R. China E-mail: wzxiecongying@163.com

*Contributed equally

Key words: radiosensitivity, esophageal cancer; microRNA-301a; WNT1; Wnt//-catenin signaling pathway directly targeting Snail and vimentin in radioresistant-ESCC cell lines; however, no inhibitory effects were exerted on Twist. Collectively, these results indicated that miR-301a increased the radiosensitivity and inhibited the migration of radioresistant-ESCC cells by targeting WNT1, thereby inactivating the Wnt/ $\beta$-catenin signaling pathway and EMT reversal. Thus, miR-301a may be a potential therapeutic target for the treatment of EC radioresistance.

\section{Introduction}

Esophageal cancer (EC) is the eighth most commonly diagnosed cancer and the sixth leading cause of cancer-associated mortality worldwide (1). Radiation therapy (RT) is a key part of multimodality EC therapy $(2,3)$. Radiotherapy resistance often leads to subsequent recurrence and metastasis $(4,5)$.

MicroRNAs (miRNAs/miRs) are a family of endogenous non-coding RNAs comprised of 18-25 nucleotides in length that function to regulate the stability of target genes by directly binding to the $3^{\prime}$ untranslated region (3' UTR) $(6,7)$. An increasing body of evidence has demonstrated that miRNAs can act as tumor promoters or suppressors to regulate various basic cellular functions (8-10). Furthermore, some studies have revealed that miRNAs directly affect radioresistance by regulating specific pathways (11), including the repair of DNA double strand breaks (12), cell cycle checkpoint activation (13), apoptosis (14) and autophagy (15). For example, Lin28-let7 regulates the radiosensitivity of human cancers through activated KRAS signaling (16).

miR-301a, located on chromosome $17 q 22-17 q 23$, is an important miRNA that has been studied in a variety of tumor types (17). miR-301a expression is upregulated in several types of cancer including pancreatic (18), prostate cancer (19) and malignant melanoma (20); it also acts as a candidate oncogene by promoting tumor proliferation and invasion. Recently, a previous study demonstrated that miR-301a promoted radioresistance in prostate cancer cells by downregulating $\mathrm{N}$-myc downstream-regulated gene 2 (NDRG2) (21). However, 
the mechanism underlying the role of miR-301a in EC radioresistance remains unclear.

In our previous study, we used a human miRNA microarray to detect the differential expression of miRNAs, comparing the human radioresistant esophageal squamous cell carcinoma (ESCC) line KYSE-150R and the parental cell line KYSE-150 (22). Based on the predicted genes and pathways of the miRNA target, the expression of miR-301a was significantly downregulated in KYSE-150R cells and it was thought to play an important role in regulating the radiosensitivity of ESCC cells. In the present study, we confirmed the effect of miR-301a on cell proliferation, epithelial-mesenchymal transition (EMT) and radiosensitivity in ESCC cells. It was revealed that miR-301a suppressed cell proliferation and migration, and enhanced radiosensitivity in ESCC cells by directly targeting WNT1.

\section{Materials and methods}

Cell culture. The human ESCC cell line KYSE-150 was purchased from the American Type Culture Collection (ATCC; Manassas, VA, USA). The radioresistant cell line KYSE-150R was previously established at our department by gradient dose irradiation treatment (22). KYSE-150 and KYSE-150R cells were maintained in RPMI-1640 medium (Gibco; Thermo Fisher Scientific, Inc., Waltham, MA, USA) with $100 \mathrm{U} / \mathrm{ml}$ of penicillin, $100 \mathrm{mg} / \mathrm{ml}$ of streptomycin and $10 \%$ fetal bovine serum (FBS; Gibco; Thermo Fisher Scientific, Inc.), in an incubator at $37^{\circ} \mathrm{C}$ containing $5 \% \mathrm{CO}_{2}$. The cell lines were sub-cultured every 2 to 3 days following digestion at room temperature with $1 \mathrm{ml} 0.25 \%$ Trypsin-EDTA (Sigma-Aldrich; Merck KGaA, Darmstadt, Germany). The viability was reported as the percentage of the number of viable cells to the total number of cells. The average viability over $95 \%$ was determined by Trypan Blue staining, at $37^{\circ} \mathrm{C}$ in an incubator containing $5 \% \mathrm{CO}_{2}$.

Cell transfection. Cultured cells were transfected with miR-301a mimic or inhibitor (Ambion; Thermo Fisher Scientific, Inc.) using Lipofectamine $2000^{\mathrm{TM}}$ reagent (Invitrogen; Thermo Fisher Scientific, Inc.) according to the manufacturer's instructions. Cells were transfected with 0,5 , 10 and $20 \mathrm{nM}$ miR-301a mimic or inhibitor or negative control (NC) for $48 \mathrm{~h}$, unless indicated otherwise.

Transwell assay. After transfection with miR-301a mimic or inhibitor, $5 \times 10^{4}$ cells were plated into the top chamber of the insert ( $8 \mu \mathrm{m}$ pore size; BD Biosciences, Franklin Lakes, NJ, USA) in medium without serum. The lower chamber was loaded with medium containing $10 \%$ FBS. The cells were incubated for $12 \mathrm{~h}$ and the cells that had migrated to the bottom of the membrane were fixed with methanol and stained with crystal violet. The number of stained cells were counted at least five random microscopic fields by a light microscope at a magnification of x200 (Olympus Corp., Tokyo, Japan).

3-(4,5-Dimethylthiazol-2-yl)-2,5-diphenyltetrazolium (MTT) assay. Cells were seeded into a 96 -well plate in triplicate at a concentration of $4 \times 10^{3}$ cells/well. Cell growth was measured by MTT bromide assay every $24 \mathrm{~h}$ from Day 1 to Day 6 . Cells were incubated with $5 \mathrm{mg} / \mathrm{ml}$ MTT for $4 \mathrm{~h}$, and subsequently solubilized in dimethyl sulfoxide (DMSO) (100 $\mu \mathrm{l} / \mathrm{well})$. The absorbance was measured at $570 \mathrm{~nm}$ using an ELISA reader.

Clonogenic assay. Cells in the exponential phase of growth were plated in triplicate onto 6 -well plates with $\sim 1 \times 10^{3}$ cells/well. These logarithmic growth cells were irradiated with doses of $0,2,4,6,8$, or 10 Gy using X-ray from a linear accelerator (Varian 2300C/D; Varian Medical Systems, Salt Lake, UT, USA) with an average dose rate of $100 \mathrm{cGy} / \mathrm{min}$. Immediately following irradiation, the cells were incubated for 10 days at $37^{\circ} \mathrm{C}$ in $5 \% \mathrm{CO}_{2}$ to allow for colony formation and then fixed with pure ethanol. Visible colonies consisting of at least 50 cells were stained with $0.5 \%$ crystal violet (Sigma-Aldrich; Merck $\mathrm{KGaA}$ ) and counted. The surviving fraction was then estimated.

Dual-luciferase reporter assay. The wild-type (wt) or mutated (mut) 3' UTR of WNT1, containing three binding sites of miR-301a, were cloned into the luciferase vector pMIR reporter. Cells were transfected with the WNT1 luciferase reporter vector, Renilla luciferase vector (Promega Corp., Madison, WI, USA) and the miRNA mimic or inhibitor using Lipofectamine $2000^{\mathrm{TM}}$ reagent (Invitrogen; Thermo Fisher Scientific, Inc.). After $48 \mathrm{~h}$, the cells were harvested, lysed and luciferase activity was assessed using the Dual-Luciferase Reporter Assay system (Promega Corp.) according to the manufacturer's protocol. Renilla luciferase was used for normalization.

Reverse transcription-quantitative polymerase chain reaction (RT-qPCR). Total RNA was extracted using TRIzol reagent (Invitrogen; Thermo Fisher Scientific, Inc.) according to the manufacturer's protocol. Total RNA $(1 \mu \mathrm{g})$ was reverse transcribed to cDNA using the SuperScript II reverse transcription kit (Thermo Fisher Scientific, Inc.). RT-qPCR was performed using SYBR-Green Master Mix (Applied Biosystems; Thermo Fisher Scientific, Inc.) and the ABI PRISM 7900 Sequence Detection System (Applied Biosystems; Thermo Fisher Scientific, Inc.). The RT-qPCR thermocycling conditions were as follows: $95^{\circ} \mathrm{C}$ for $10 \mathrm{~min}$, followed by 40 cycles at $95^{\circ} \mathrm{C}$ for $15 \mathrm{sec}$ and $60^{\circ} \mathrm{C}$ for $1 \mathrm{~min}$. $\beta$-actin was used as an internal control and the fold-changes of target genes were calculated using the $2^{-\Delta \Delta \mathrm{Cq}}$ method (23). Primer sequences of Wnt pathway- and EMT-associated genes are presented in Table I.

Western blot analysis. Proteins were extracted from cultured cells using RIPA buffer (Thermo Fisher Scientific, Inc). Protein concentration was determined using the BCA protein assay kit (Thermo Fisher Scientific, Inc.). Lysates containing $100 \mu \mathrm{g}$ of protein were mixed with loading buffer containing $5 \% \beta$-mercaptoethanol. Extracted proteins were subjected to $10 \%$ SDS-PAGE and transferred to PVDF membranes $(0.45 \mu \mathrm{m})$. The membranes were then incubated with $5 \%$ non-fat dry milk in phosphate-buffered saline (PBS) for $1 \mathrm{~h}$ at room temperature to block non-specific binding sites. Then, the membranes were incubated with primary antibodies overnight at $4^{\circ} \mathrm{C}$, followed by a secondary antibody (1:20,000; anti-rabbit or anti-mouse IgG, HRP-linked antibody, cat. nos. 7071 and 7072, respectively; Cell Signaling 
Table I. Primer sequences of Wnt pathway- and EMT-associated genes.

\begin{tabular}{lll}
\hline Target gene & \multicolumn{1}{c}{ Forward primer $\left(5^{\prime}-3^{\prime}\right)$} & Reverse primer $\left(5^{\prime}-3^{\prime}\right)$ \\
\hline WNT1 & TAAGCAGGTTCGTGGAGGAG & GGTTTCTGCTACGCTGCTG \\
E-cadherin & GACCGGTGCAATCTTCAAA & TTGACGCCGAGAGCTACAC \\
Twist & TCCATTTTCTCCTTCTCTGGAA & CCTTCTCGGTCTGGAGGAT \\
Vimentin & GCAAAGATTCCACTTTGCGT & GAAATTGCAGGAGGAGATGC \\
Snail & TCTGAGTGGGTCTGGAGGTG & CTCTAGGCCCTGGCTGCTAC \\
TCF4 & CATAGGGAGTCCCATCTCCA & GGACCAACTTCTTTGGCAAG \\
Cyclin D1 & GGCGGATTGGAAATGAACTT & TCCTCTCCAAAATGCCAGAG \\
$\beta$-actin & AAAGACCTGTACGCCAACAC & GTCATACTCCTGCTTGCTGAT
\end{tabular}

Technology, Inc.) incubation for $2 \mathrm{~h}$ at room temperature. The protein bands were visualized with the SuperSignal West Pico Chemiluminescent kit (Pierce; Thermo Fisher Scientific, Inc.). The following primary antibodies were used: Cyclin D1 (1:1,000; cat. no. 2978), E-cadherin (1:1,000; cat. no. 3195), $\beta$-catenin $(1: 1,000$; cat. no. 8480$)$ and Snail $(1: 1,000$; cat. no. 3879; all from CST Biological Reagents Co., Ltd., Shanghai, China), WNT1 (1:1,000; cat. no. ab15251; Abcam, Cambridge, MA, USA) and vimentin (1:1,000; cat. no. 5741; CST Biological Reagents Co., Ltd.) and Tubulin (1:4,000; cat. no. T5168; Sigma-Aldrich; Merck KGaA).

Immunofluorescence staining. Cells were fixed with $4 \%$ formaldehyde, permeabilized with $0.5 \%$ Triton, and then incubated with an E-cadherin (1:500) or vimentin (1:500) antibody overnight. After washing with PBS, the cells were incubated with Alexa Fluor 488-labeled secondary antibody (1,000-fold dilution; cat. no. 34201; Invitrogen; Thermo Fisher Scientific, Inc.) for $45 \mathrm{~min}$. Cells were counterstained with DAPI to label the nuclei. Images were captured with a Nikon fluorescent microscope.

Statistical analysis. Data were expressed as the mean \pm standard deviation from at least three independent experiments. Student's t-test was used to compare the differences between two groups. All statistical analyses were performed using SPSS 15.0 software (SPSS, Inc., Chicago, IL, USA). P<0.05 was considered to indicate a statistically significant difference.

\section{Results}

miR-301a inhibits cell proliferation and migration, and increases radioresistant cell sensitivity to irradiation in ESCC. The present study initially transfected KYSE-150R cells with the miR-301a mimic in order to upregulate miR-301a in vitro $(\mathrm{P}<0.01$; Fig. $1 \mathrm{~A})$. The overexpression of miR-301a in KYSE-150R cells decreased cell viability when compared with cells transfected with $\mathrm{NC}$ mimic cells $(\mathrm{P}<0.05$; Fig. 1B). The results of the colony formation assays revealed that overexpressing miR-301a significantly enhanced the effects of radiotherapy in KYSE-150R cells ( $\mathrm{P}<0.05$; Fig. 1C). Upregulation of miR-301a in KYSE-150R cells led to a 30-40\% reduction in the levels of migration when compared with the NC-mimic cells ( $\mathrm{P}<0.01$; Fig. 1D).
Next, the present study suppressed the expression of miR-301a in KYSE-150 cells via transfection with a miR-301a inhibitor ( $\mathrm{P}<0.05$; Fig. 2A). The miR-301a inhibitor groups exhibited enhanced cell proliferation when compared with the NC inhibitor group ( $\mathrm{P}<0.05$; Fig. 2B). The KYSE-150 cells with reduced miR-301a levels had a significantly greater survival fraction when compared with the NC cells $(\mathrm{P}<0.05$; Fig. 2C), indicating that these cells had decreased radiosensitivity. Anti-miR301a cells displayed significantly higher migration potential, compared to the NC group ( $\mathrm{P}<0.01$; Fig. 2D).

miR-301a directly targets the coding region of WNT1. Next the present study evaluated the potential targets of miR-301a. Bioinformatics analysis was performed to identify potential candidate targets of miR-301a using publicly available algorithms. As revealed in Fig. 3A, the 3' UTR WNT1 contained the target sequence of miR-301a. Subsequently, RT-qPCR and western blotting were employed to determine the ability of miR-301a to regulate the expression of WNT1. Overexpression of miR-301a markedly downregulated the mRNA and protein levels of WNT1 in KYSE-150R cells. Conversely, inhibition of miR-301a markedly increased the expression of WNT1 at the mRNA and protein levels in the KYSE-150 group (Fig. 3B and C).

To further explore whether miR-301a could interact with the 3' UTR of WNT1, the present study performed luciferase reporter assays. As indicated in Fig. 3D, the miR-301a mimic significantly inhibited luciferase activity in cells expressing pMIR-WNT1-wt 3' UTR. However, co-transfection with the pMIR-WNT1-mut plasmid and the miR-301a mimic did not significantly alter the luciferase activity when compared with the NC group $(\mathrm{P}>0.05)$. Collectively, these results demonstrated that WNT1 is directly targeted by miR-301a.

Comparisons between the expression of Wnt/ $\beta$-catenin signaling pathway-related genes and proteins among the different groups. RT-qPCR and western blot analyses were conducted to investigate whether miR-301a expression could markedly affect the expression of $\mathrm{Wnt} / \beta$-catenin-related proteins. As revealed in Fig. 4A, miR-301a mimic-transfected cells (at the greatest concentration of $20 \mathrm{nM}$ ) had significantly lower mRNA expression of transcription factor 4 (TCF4) and cyclin D1 when compared to the KYSE-150R control groups. In miR-301a inhibitor-transfected cells, TCF4 and cyclin D1 were significantly increased. Furthermore, the 

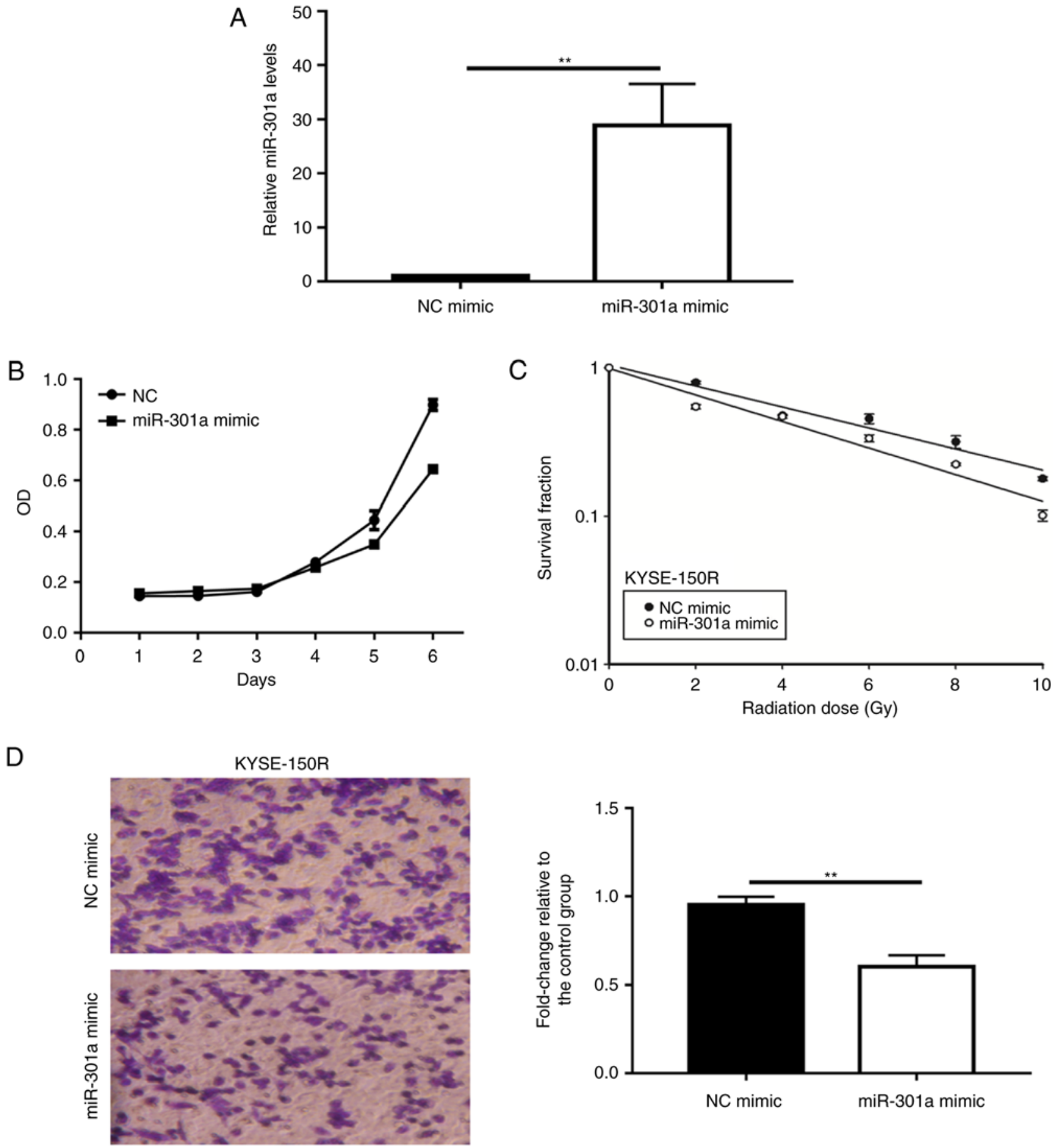

Figure 1. Upregulation of miR-301a in KYSE-150R cells inhibits cell proliferation and migration and increases cell sensitivity to irradiation. (A) miR-301a was markedly upregulated in KYSE-150R cells transfected with the miR-301a mimic. (B) The proliferation rate of KYSE-150R cells overexpressing miR-301a mimics was assessed by MTT assay. (C) A clonogenic survival assay was performed to determine the effect of miR-301a upregulation on the radiosensitivity of KYSE-150R cells. (D) Cell Transwell assays were conducted to investigate the effect of miR-301a on the migration ability of KYSE-150R resistant cells. ${ }^{* *} \mathrm{P}<0.01$. NC, negative control; OD, optical density; miR, microRNA.

protein expression of $\beta$-catenin and cyclin D1 was reduced in the miR-301a mimic-transfected group. However, the miR-301a inhibitor-transfected group exhibited the opposite results $(\mathrm{P}<0.05$; Fig. 4B).

miR-301a affects EMT-related protein expression. Our previous study demonstrated that EMT mediates radioresistance in human ESCC cells (24). Therefore, in the present study classic EMT biomarkers and transcription factors were detected in the different cell groups. The results of the RT-qPCR assay and western blotting revealed that the gene and protein expression levels of E-cadherin in KYSE-150 cells transfected with miR-301a inhibitors were decreased than those observed in the NC groups, while E-cadherin expression in the miR-301a-mimic groups was significantly increased when compared with the NC groups (Fig. 5A and B). Furthermore, 

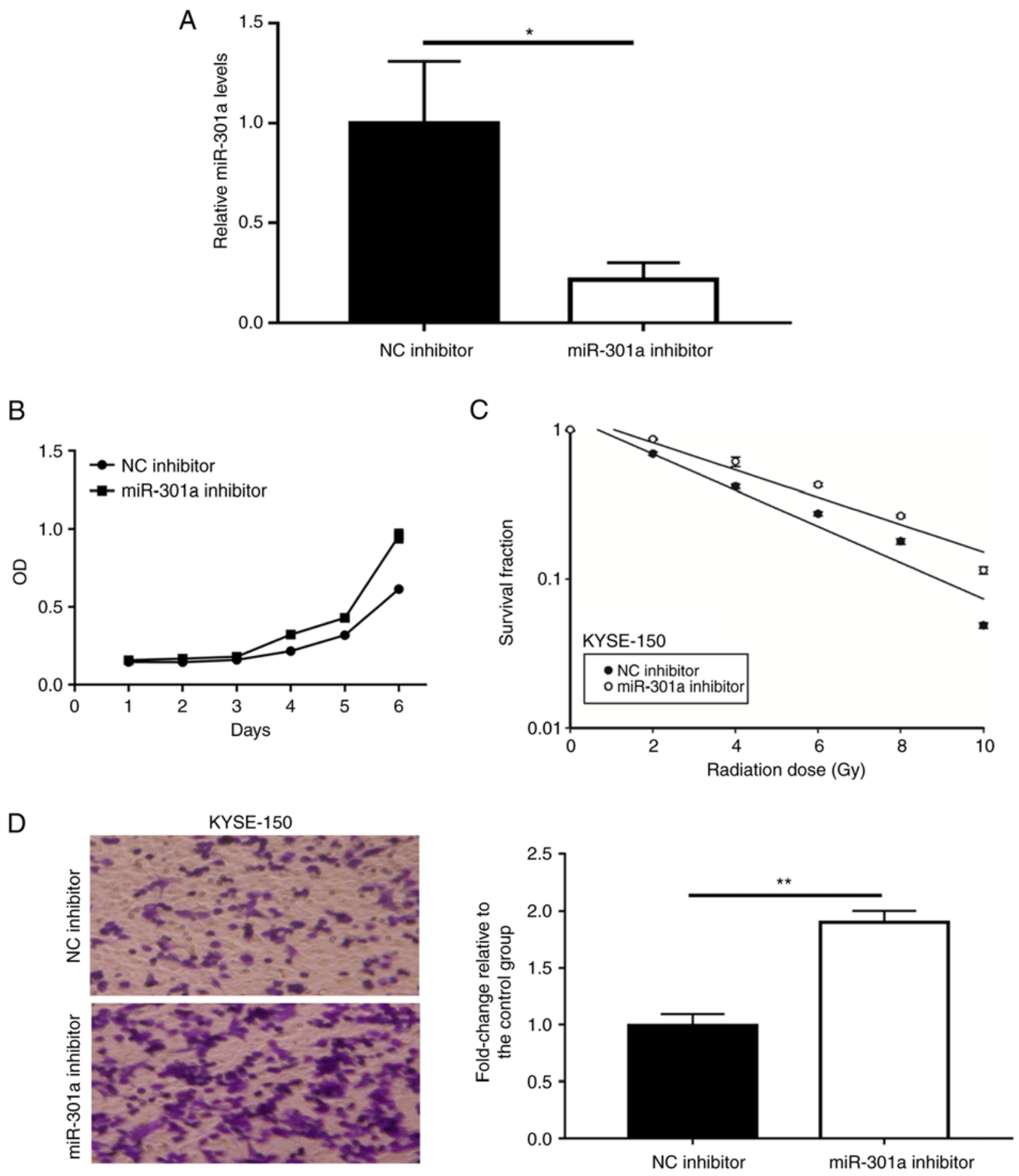

Figure 2. Downregulation of miR-301a enhances cell proliferation, decreases radiosensitivity and promotes cell migration in KYSE-150 cells. (A) miR-301a was markedly downregulated in KYSE-150 cells transfected with the miR-301a inhibitor. (B) Cell proliferative ability was evaluated following transfection with miR-301a inhibitor or NC inhibitor by MTT assay. (C) Colony formation assays were conducted using KYSE-150 cells transfected with miR-301a inhibitor or NC inhibitor. (D) The effect of miR-301a inhibitors on the migration of KYSE-150 cells was evaluated by a cell migration assay. ${ }^{*} \mathrm{P}<0.05$; ${ }^{* *} \mathrm{P}<0.01$. NC, negative control; OD, optical density; miR, microRNA.

expression of vimentin and Snail in the miR-301a-inhibitor group was upregulated, whereas the expression of vimentin and Snail was downregulated in the miR-301a-mimic group. However, the regulation of miR-301a did not significantly affect Twist expression ( $\mathrm{P} \geq 0.05$; Fig. 5A and $\mathrm{B}$ ).

Immunofluorescence analysis was conducted to confirm the localization of vimentin and E-cadherin in the miR-301a overexpression and knockout groups. Intense staining in the cell membrane indicated that E-cadherin was shuttled to the cell membrane in miR-301a-overexpressing cells, while less staining in knockout miR-301a cells indicated that E-cadherin was downregulated (Fig. 5C). Conversely the expression of vimentin in the cell membrane of miR-301a mimic-transfected cells was reduced. These results indicated that miR-301a may be an upstream regulator of E-cadherin and vimentin expression, and an inducer of EMT phenotypes. 


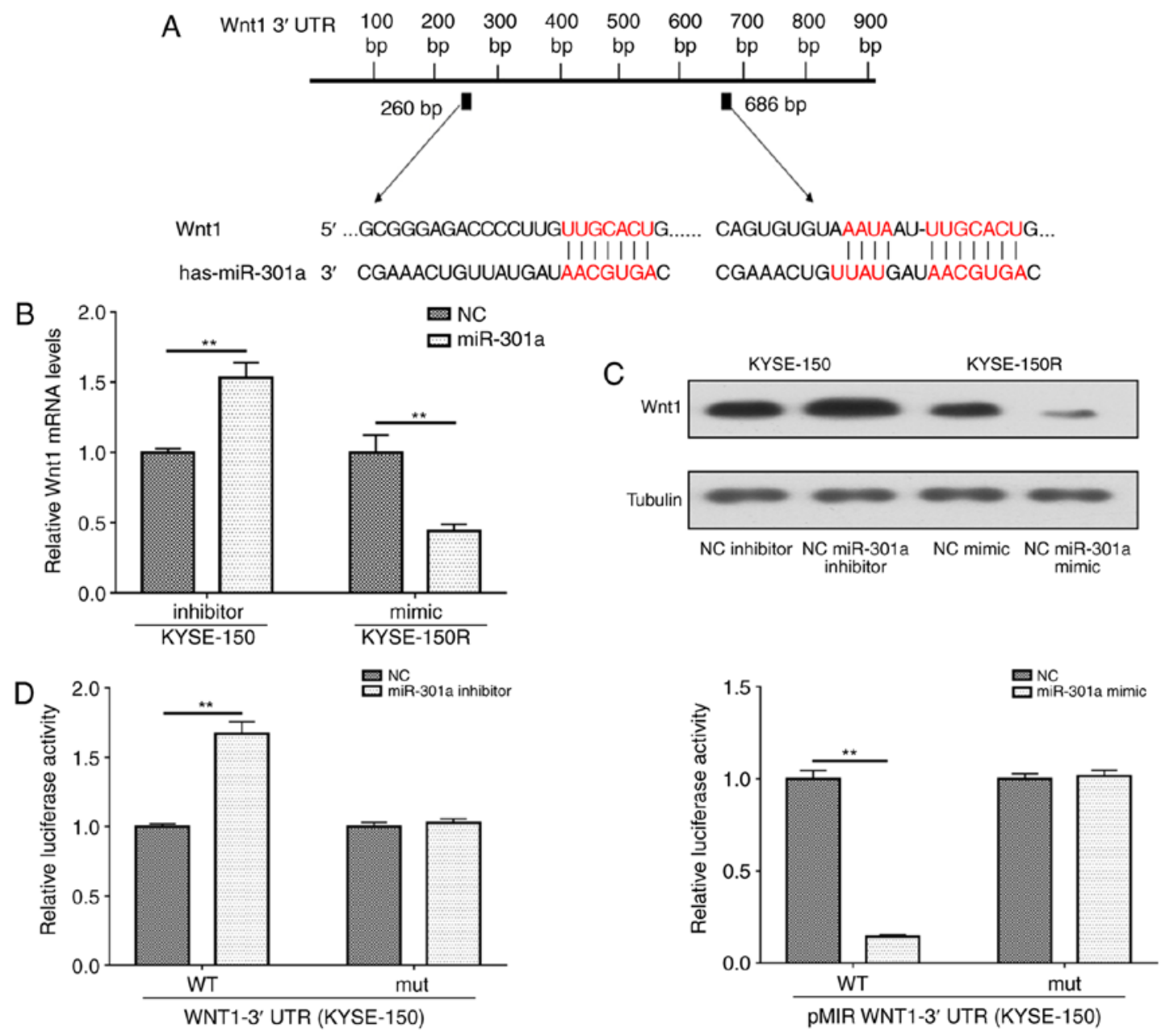

Figure 3. miR-301a directly targets the coding region of WNT1. (A) The sequences of the 3'UTR of WNT1 predicted to bind to miR-301a. (B) Reverse transcription-quantitative polymerase chain reaction was performed to determine WNT1 mRNA expression in the KYSE-150R or KYSE-150 cells transfected with miR-301a mimics or inhibitor. (C) Western blotting was conducted to determine WNT1 protein expression in the KYSE-150R or KYSE-150 cells transfected with miR-301a mimics or inhibitor. (D) Relative luciferase activities of the luciferase reporter plasmids in KYSE-150R or KYSE-150 cells transfected with miR-301a mimics or inhibitor. ${ }^{* * *} \mathrm{P}<0.01$ vs. NC inhibitor. mut, mutant; wt, wild-type; NC, negative control; UTR, untranslated region.

A
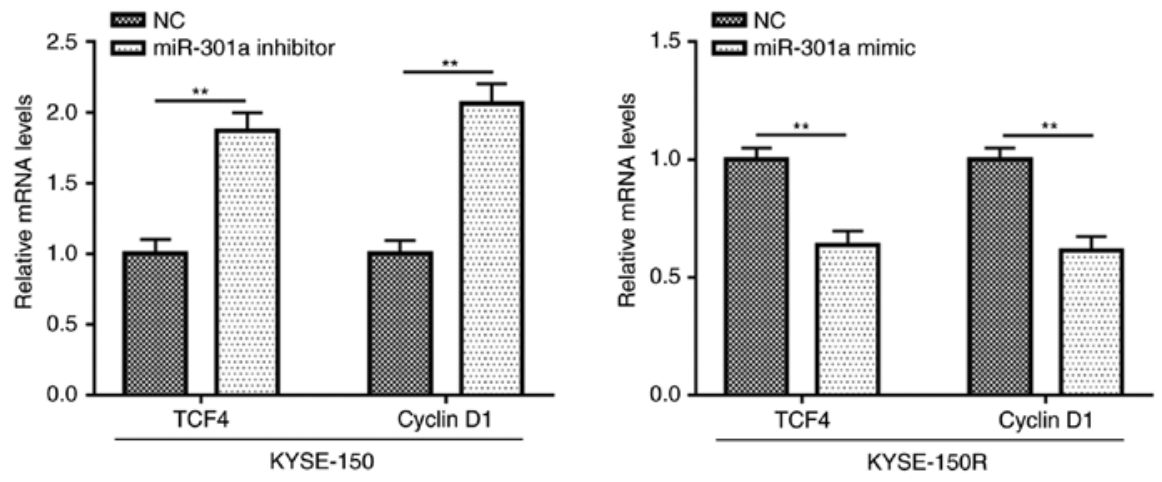

B
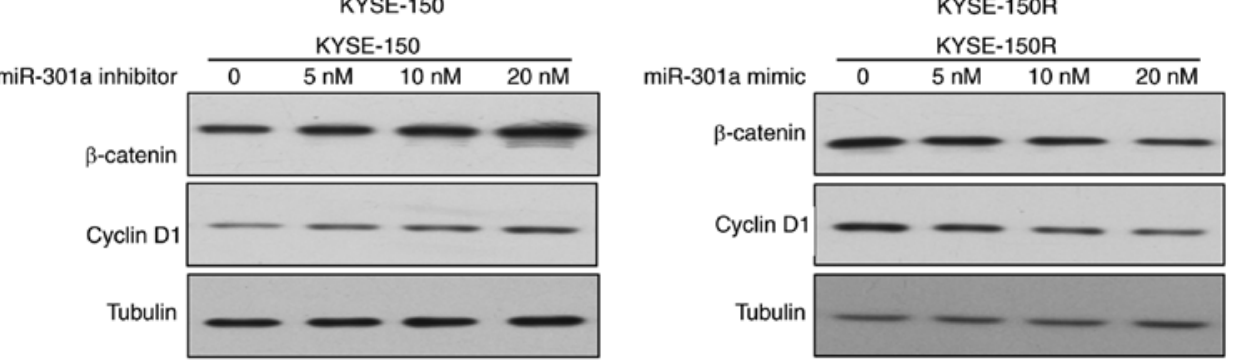

Figure 4.Comparisons of the expression of proteins associated with the Wnt/ $\beta$-catenin signaling pathway in the different groups. (A) Reverse transcription-quantitative polymerase chain reaction was performed to determine the mRNA expression of TCF4 and cyclin D1 in the different groups. (B) Western blotting was conducted to determine $\beta$-catenin and cyclin D1 protein expression in the different groups. ${ }^{* *} \mathrm{P}<0.01$ vs. the respective control. TCF4, transcription factor 4. 


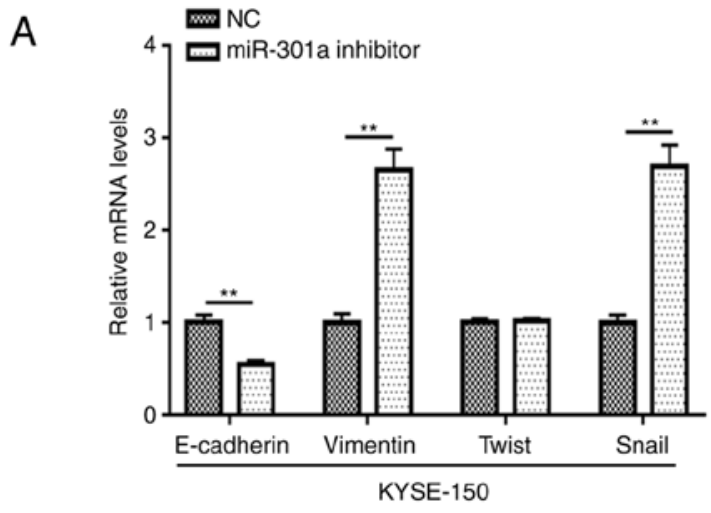

B

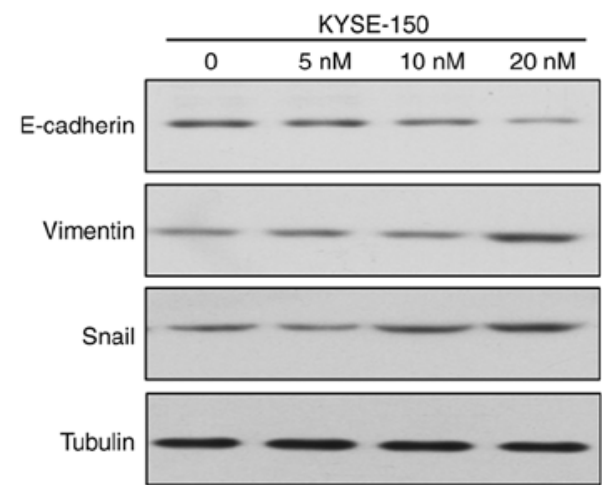

C

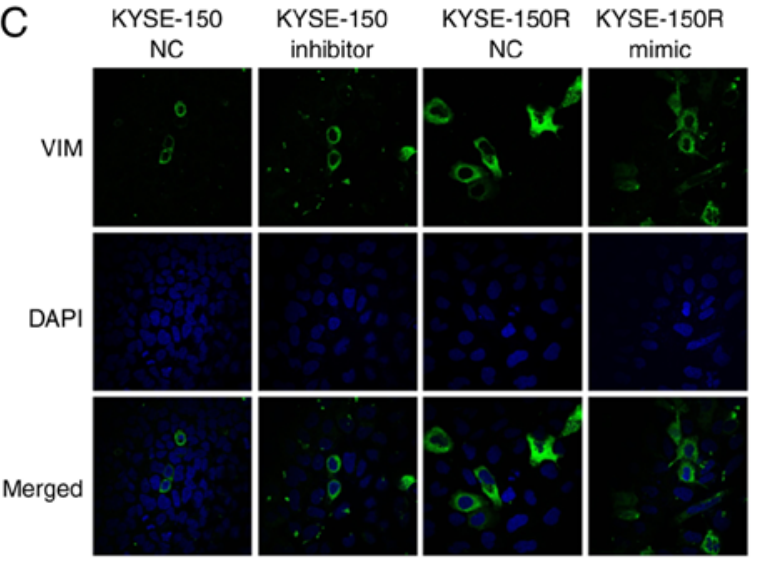

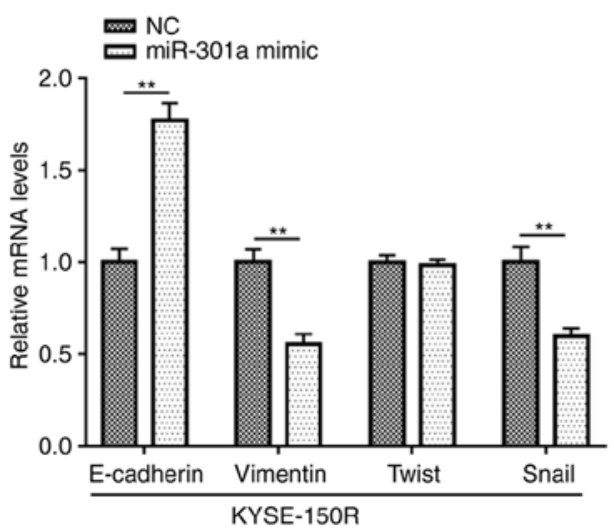
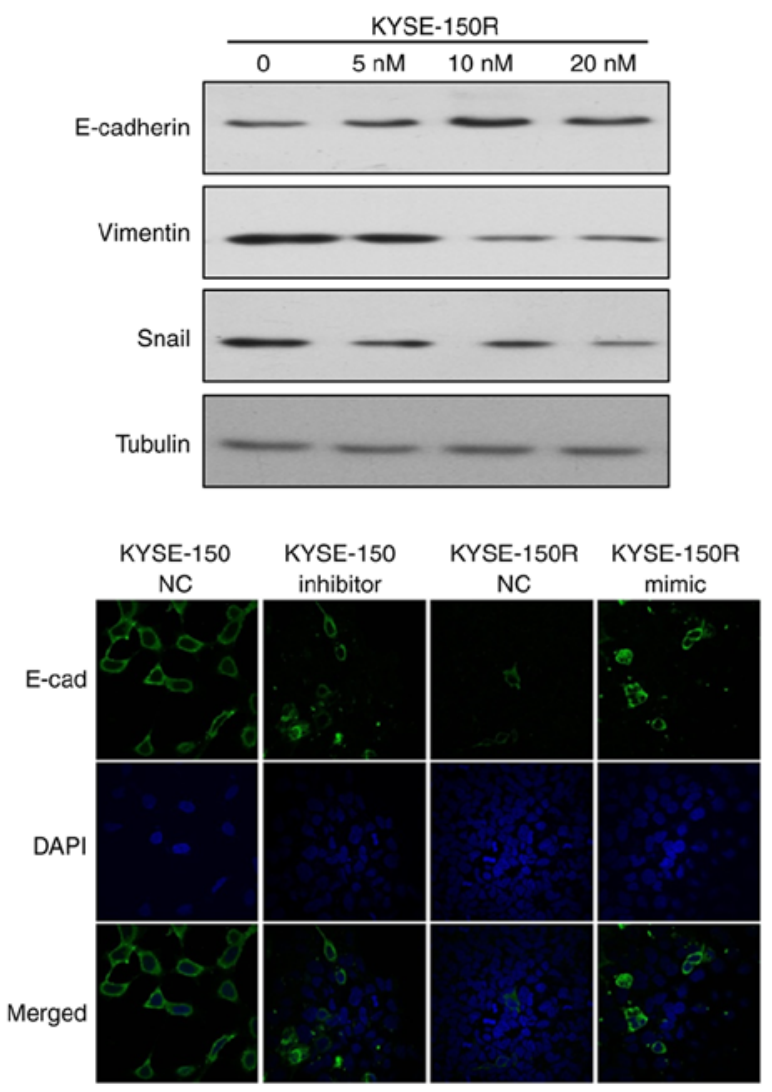

Figure 5. miR-301a affects EMT-related protein expression. (A) The relative expression of EMT-TFs in the different groups was analyzed by reverse transcription-quantitative polymerase chain reaction. (B) Cells were analyzed by western blot assays to determine the protein expression of EMT-TFs. (C) Expression of E-cadherin and vimentin was detected by immunofluorescence analysis. Nuclei were counterstained with DAPI (blue). ${ }^{* *} \mathrm{P}<0.01$ vs. the respective control. EMT, epithelial-mesenchymal transition; TF, transcription factor.

\section{Discussion}

Acquired radioresistance is considered to be an important cause of treatment failure in EC patients. Our previous study revealed that miR-301a was a candidate in the aberrant profile of radiosensitive miRNAs and was associated with ESCC radiosensitivity (22). However, there is limited research on its function. In the present study, miR-301a was revealed to regulate the radiosensitivity of ESCC by targeting WNT1. The results indicated that miR-301a may be a new type of radiosensitivity-related miRNA for future EC radioresistant therapeutic strategies.

miR-301a has been identified as a candidate oncogene in different types of cancers. In colorectal cancer, ectopic
miR-301a expression was observed to promote migration and invasion by downregulating transforming growth factor $\beta$ receptor (17). Nam et al (25) demonstrated that miR-301a played an oncogenic role in prostate cancer by directly targeting the p63 tumor suppressor. Kawano et al (26) demonstrated that restoration of miR-301a downregulation decreased ESC proliferation and tumorigenesis, and that the oncogenic activity may involve the negative regulation of the target gene, phosphatase and tensin homolog. In the present study, upregulation of miR-301a in KYSE-150R cells was revealed to inhibit cell proliferation and migration, and increase cell sensitivity to irradiation. Furthermore, inhibition of miR-301a in KYSE-150 cells reduced cell sensitivity to irradiation. However, miR-301a function in the present study was markedly different to that 
reported in previous studies. This may be due to the fact that the different functions of miRNAs depend on the particular type of cancer cell line used because of its particular genetic background.

miRNAs exert their biological functions by binding with the 3' UTR of their target genes. Wang et al (21) observed that higher expression levels of miR-301a and miR-301b resulted in elevated autophagy and increased radioresistance in $\mathrm{LNCaP}$ cells by targeting NDRG2. The present study confirmed that WNT1 was a target of miR-301a based on the results of the luciferase reporter assays. In agreement with this, the present study also revealed that miR-301a overexpression significantly inhibited WNT1 expression at both the protein and mRNA levels, indicating that miR-301a suppressed ESCC cell activities by targeting WNT1.

Numerous previous studies have focused on WNT1, a major member of $\mathrm{Wnt} / \beta$-catenin signaling, and have often reported that it is altered in EC. The Wnt/ $\beta$-catenin signaling pathway plays a crucial role in EC biological processes, including cell proliferation, apoptosis, and motility. Recently, previous research has reported that aberrant activation of canonical Wnt/ $\beta$-catenin signaling after long-term exposure to fractionated irradiation was associated with the development of radioresistance in many types of human cancers. In our pervious study, significantly upregulated expression levels of WNT1, catenin $\beta 1$ and cyclin D1 were observed in the radioresistant ESCC KYSE-150R cell line, indicating that the Wnt/ $\beta$-catenin signaling pathway was activated (24). As crucial biological regulators, miRNAs inhibit targets of signaling pathways and can promote or inhibit cancer progression in a context- or target-dependent manner. (27). miR-185-3p regulated nasopharyngeal carcinoma radioresistance by targeting WNT2B (28). The present study revealed that $\beta$-catenin and cyclin D1 may be key molecules in the Wnt/ $\beta$-catenin signaling pathway, as their expression was altered when miR-301a was upregulated. These studies provide the basis for the future exploration of the potential mechanisms underlying miR-301a functions and how WNT1 is involved in EC radiation resistance.

Notably, the present study demonstrated that miR-301a not only enhanced radiosensitivity but also affected EMT by changing vimentin and E-cadherin expression. EMT is a vitally important biological process during which epithelial cells lose their polarity and change to a mesenchymal phenotype (29). Numerous studies have demonstrated that EMT plays an important role in cancer malignant behaviors, including radiation resistance (30), drug resistance (31) and cancer stem cells (32). The epithelial marker E-cadherin and the mesenchymal marker vimentin are often used as markers of EMT during metastatic progression. Our previous study reported that the ESCC cell line KYSE-150R presented with typical morphological changes of the EMT phenotype with significantly decreased E-cadherin and increased vimentin expression. The results of the present study revealed that miR-301a and WNT1 affected the expression of EMT-related biomolecules, indicating that miR-301a and WNT1 may induce EC radioresistance through EMT.

However, there are some limitations in our study. For example, we only used one radioresistant cell line to conduct the study. In vivo models will be set up to define the roles of
miR-301a in EC radioresistance in further research. In conclusion, upregulation of miR-301a inactivated the Wnt/ $\beta$-catenin signaling pathway by targeting WNT1, leading to increased radiosensitivity and reduced migration of cancer cells. In addition, targeting this novel miR-301a/Wnt/ $\beta$-catenin axis may be a promising strategy for the future treatment of EC radioresistance.

\section{Acknowledgements}

Not applicable.

\section{Funding}

The present study was supported by the Natural Science Foundation of Zhejiang Province (grant nos. LQ15H160013, LY17H160051 and LY15H280013) and the Natural Science Foundation of China (grant no. 81602658).

\section{Availability of data and materials}

The datasets used during the present study are available from the corresponding author upon reasonable request.

\section{Authors' contributions}

MC designed the experiments. HS, YW, LS, and ZF performed the biological experiments. MC, YW, LS and HS provided administrative support and funded the experiments. HS, YF, ZF and CX analyzed the data. HS, YF, CX and MC drafted the manuscript. All authors read and approved the manuscript and agree to be accountable for all aspects of the research in ensuring that the accuracy or integrity of any part of the work are appropriately investigated and resolved.

\section{Ethics approval and consent to participate}

Not applicable.

\section{Patient consent for publication}

Not applicable.

\section{Competing interests}

The authors declare that they have no competing interests.

\section{References}

1. Napier KJ, Scheerer M and Misra S: Esophageal cancer: A Review of epidemiology, pathogenesis, staging workup and treatment modalities. World J Gastrointest Oncol 6: 112-120, 2014.

2. Fokas E, Weiss $C$ and Rödel C: The role of radiotherapy in the multimodal management of esophageal cancer. Dig Dis 31: 30-37, 2013.

3. Sjoquist KM, Burmeister BH, Smithers BM, Zalcberg JR, Simes RJ,Barbour A, Gebski V and Australasian Gastro-Intestinal Trials Group: Survival after neoadjuvant chemotherapy or chemoradiotherapy for resectable oesophageal carcinoma: An updated meta-analysis. Lancet Oncol 2: 681-692, 2011.

4. Barker HE, Paget JT, Khan AA and Harrington KJ: The tumour microenvironment after radiotherapy: Mechanisms of resistance and recurrence. Nat Rev Cancer 15: 409-425, 2015. 
5. Maier P, Hartmann L, Wenz F and Herskind C: Cellular pathways in response to ionizing radiation and their targetability for tumor radiosensitization. Int J Mol Sci 17. pii: E102, 2016.

6. Lim LP, Lau NC, Garrett-Engele P, Grimson A, Schelter JM, Castle J, Bartel DP, Linsley PS and Johnson JM: Microarray analysis shows that some microRNAs downregulate large numbers of target mRNAs. Nature 433: 769-773, 2005.

7. Bartel DP: MicroRNAs: Target recognition and regulatory functions. Cell 136: 215-233, 2009.

8. Garzon R, Marcucci G and Croce CM: Targeting microRNAs in cancer: Rationale, strategies and challenges. Nat Rev Drug Discov 9: 775-778, 2010.

9. Hummel R, Hussey DJ and Haier J: MicroRNAs: Predictors and modifiers of chemo- and radiotherapy in different tumour types. Eur J Cancer 46: 298-311, 2010.

10. Farazi TA, Spitzer JI, Morozov P and Tuschl T: miRNAs in human cancer. J Pathol 223: 102-115, 2011.

11. Zhao L, Lu X and Cao Y: MicroRNA and signal transduction pathways in tumor radiation response. Cell Signal 25: 1625-1634, 2013.

12. Hu B, Wang X, Hu S, Ying X, Wang P, Zhang X, Wang J, Wang H and Wang Y: miR-21-mediated radioresistance occurs via promoting repair of DNA double strand breaks. J Biol Chem 292: $3531-3540,2017$

13. Bhattacharya A, Schmitz U, Wolkenhauer O, Schönherr M, Raatz Y and Kunz M: Regulation of cell cycle checkpoint kinase WEE1 by miR-195 in malignant melanoma. Oncogene 32 3175-3183, 2013.

14. Wang S, Pan Y,Zhang R, Xu T, Wu W, Zhang R, Wang C, Huang H, Calin CA, Yang H and Claret FX: Hsa-miR-24-3p increases nasopharyngeal carcinoma radiosensitivity by targeting both the 3'UTR and 5'UTR of Jab1/CSN5. Oncogene 35: 6096-6108, 2016

15. Wang P, Zhang J, Zhang L, Zhu Z, Fan J, Chen L, Zhuang L, Luo J, Chen H, Liu L, et al: MicroRNA 23b regulates autophagy associated with radioresistance of pancreatic cancer cells Gastroenterology 145: 1133-1143, 2013.

16. Oh JS, Kim JJ, Byun JY and Kim IA: Lin28-let7 modulates radiosensitivity of human cancer cells with activation of K-Ras. Int J Radiat Oncol Biol Phys 76: 5-8, 2010.

17. Zhang W, Zhang T, Jin R, Zhao H, Hu J, Feng B, Zang L, Zheng M and Wang M: MicroRNA-301a promotes migration and invasion by targeting TGFBR2 in human colorectal cancer. J Exp Clin Cancer Res 33: 113, 2014.

18. Xia X, Zhang K, Luo G, Cen G, Cao J, Huang K and Qiu Z: Downregulation of miR-301a-3p sensitizes pancreatic cancer cells to gemcitabine treatment via PTEN. Am J Transl Res 9: 1886-1895, 2017.
19. Damodaran C, Das TP, Papu John AM, Suman S, Kolluru V, Morris TJ, Faber EN, Rai SN, Messer JC, Alatassi H, et al: miR-301a expression: A prognostic marker for prostate cancer. Urol Oncol 34: e13-e20, 2016.

20. Cui L, Li Y, Lv X, Li J, Wang X, Lei Z and Li X: Expression of microRNA-301a and its functional roles in malignant melanoma. Cell Physiol Biochem 40: 230-244, 2016.

21. Wang W, Liu M, Guan Y and Wu Q: Hypoxia-responsive miR-301a and miR-301b promote radioresistance of prostate cancer cells via downregulating NDRG2. Med Sci Monit 22: 2126-2132, 2016

22. Su H, Jin X, Zhang X, Xue S, Deng X, Shen L, Fang Y and Xie C: Identification of microRNAs involved in the radioresistance of esophageal cancer cells. Cell Biol Int 38: 318-325, 2014.

23. Livak KJ and Schmittgen TD: Analysis of relative gene expression data using real-time quantitative PCR and the $2^{-\Delta \Delta C_{\mathrm{T}}}$ method. Methods 25: 402-408, 2001.

24. Su H, Jin X, Zhang X, Zhao L, Lin B, Li L, Fei Z, Shen L, Fang Y, Pan H, et al: FH535 increases the radiosensitivity and reverses epithelial-to-mesenchymal transition of radioresistant esophageal cancer cell line KYSE-150R. J Transl Med 13: 104, 2015.

25. Nam RK, Benatar T, Wallis CJ, Amemiya Y, Yang W, Garbens A, Naeim M, Sherman C, Sugar L and Seth A: MiR-301a regulates E-cadherin expression and is predictive of prostate cancer recurrence. Prostate 76: 869-884, 2016.

26. Kawano M, Tanaka K, Itonaga I, Iwasaki $\mathrm{T}$ and Tsumura $\mathrm{H}$ : MicroRNA-301a promotes cell proliferation via PTEN targeting in Ewing's sarcoma cells. Int J Oncol 48: 1531-1540, 2016.

27. Song JL, Nigam P, Tektas SS and Selva E: microRNA regulation of Wnt signaling pathways in development and disease. Cell Signal 27: 1380-1391, 2015

28. Li G, Wang Y, Liu Y, Su Z, Liu C, Ren S, Deng T, Huang D, Tian Y and Qiu Y: miR-185-3p regulates nasopharyngeal carcinoma radioresistance by targeting WNT2B in vitro. Cancer Sci 105: 1560-1568, 2014.

29. Mani SA, Guo W, Liao MJ, Eaton EN, Ayyanan A, Zhou AY, Brooks M, Reinhard F, Zhang CC, Shipitsin M, et al: The epithelial-mesenchymal transition generates cells with properties of stem cells. Cell 133: 704-715, 2008.

30. Marie-Egyptienne DT, Lohse I and Hill RP: Cancer stem cells, the epithelial to mesenchymal transition (EMT) and radioresistance: Potential role of hypoxia. Cancer Lett 341: 63-72, 2013.

31. Shibue T and Weinberg RA: EMT, CSCs, and drug resistance: The mechanistic link and clinical implications. Nat Rev Clin Oncol 14: 611-629, 2017

32. Martin-Belmonte F and Perez-Moreno M: Epithelial cell polarity, stem cells and cancer. Nat Rev Cancer 12: 23-38, 2011. 